\title{
Improving the Efficiency of Medical Services Systems: A New Integrated Mathematical Modeling Approach
}

\author{
Davood Shishebori and Mohammad Saeed Jabalameli \\ Department of Industrial Engineering, Iran University of Science and Technology, Tehran 1684613114, Iran \\ Correspondence should be addressed to Davood Shishebori; d2005shishehbori@gmail.com
}

Received 27 January 2013; Accepted 5 May 2013

Academic Editor: Ming Li

Copyright ( 2013 D. Shishebori and M. S. Jabalameli. This is an open access article distributed under the Creative Commons Attribution License, which permits unrestricted use, distribution, and reproduction in any medium, provided the original work is properly cited.

\begin{abstract}
Nowadays, the efficient design of medical service systems plays a critical role in improving the performance and efficiency of medical services provided by governments. Accordingly, health care planners in countries especially with a system based on a National Health Service (NHS) try to make decisions on where to locate and how to organize medical services regarding several conditions in different residence areas, so as to improve the geographic equity of comfortable access in the delivery of medical services while accounting for efficiency and cost issues especially in crucial situations. Therefore, optimally locating of such services and also suitable allocating demands them, can help to enhance the performance and responsiveness of medical services system. In this paper, a multiobjective mixed integer nonlinear programming model is proposed to decide locations of new medical system centers, link roads that should be constructed or improved, and also urban residence centers covered by these medical service centers and link roads under investment budget constraint in order to both minimize the total transportation cost of the overall system and minimize the total failure cost (i.e., maximize the system reliability) of medical service centers under unforeseen situations. Then, the proposed model is linearized by suitable techniques. Moreover, a practical case study is presented in detail to illustrate the application of the proposed mathematical model. Finally, a sensitivity analysis is done to provide an insight into the behavior of the proposed model in response to changes of key parameters of the problem.
\end{abstract}

\section{Introduction}

In recent years, the design of medical services (MS) systems has attracted significant attention by different scientific societies. Providing an adequate service level is an attempt for many MS systems, but it is of special concern in the context of MS centers, location and also link constructing or improving. Despite the recent progress in accessibility, answer improvement to customers, availability of more advanced equipments, some pressing problems are worth to be further investigated like the reduction of the investment costs for locating MS centers, constructing or improving of link roads, and also transportation costs of customers, to mention a few. Recently, MS systems in most countries in the world endeavor primarily to maximize the populations' health, equity, efficiency, and quality, and, at a second level, to control and/or minimize different health care costs. In addition, as a third level, the reliability of the designed MS system is very important and a robust and also efficient MS systems can significantly affect the provided medical services for populations of a region especially in crisis situations. But considering the reliability of MS systems makes the design and the management of efficient MS systems very difficult and merits specific attention by the health care planners.

In order to meet these objectives, governments need to have efficient and accurate plans for locating, allocating, and improving MS systems. Accordingly, several related decisions as follows need to be made: where should MS centers be located so as to improve the geographic equity of access? What is the optimal structure of the MS centers network? How should the link network be constructed or improved? What are the costs required to improve access? And are they acceptable? How should the design of MS systems be changed to increase the reliability of MS systems? How much should the extra spending have to improve the reliability of MS systems? [1]. 
Such strategic decisions, about locating MS centers and also organizing MS systems networks with respect to the different actual and practical conditions have suitable and efficient tradeoffs to follow the above objectives, such as the tradeoff between equity, efficiency, and costs [2]. For example, enhancing the geographic equity of access might mention building small MS centers close to populations, which leads to inefficiencies in scale and higher costs.

On the other hand, the significant cost of some medical equipment and the poor availability of highly skilled human or particular drug resources (such as specialized doctors or special drugs) might imply that the supply of services is delivered to large populations, which might have an undesired impact on geographic access. As another example, reducing the costs of locating $\mathrm{MS}$ centers and also allocating demands to them may cause the increase of the failure costs of MS centers. Also, excessive increasing in costs of locating and allocating of MS centers to reduce the failure costs and subsequently increase the reliability of MS systems may be uneconomic and not possible for most governments. So an accurate and efficient planning and modeling of locating MS centers and allocating them can be caused to an enough confidant and practical approach to reach the mentioned objectives.

Some researchers have focused their efforts on the strategic planning, others on tactical support (including routing of MS vehicles for nonurgent calls) and on operational control (MS vehicles dispatch and relocation). Although some improvement areas can be identified, successful researches should proceed for stronger movements from the strategic level to the operational one [3]. This paper focuses on the strategic level and aims to propose a new mathematical model that can act as a support tool to help MS systems planners and improve the geographic equity of access and reduce transportation costs and also failure costs of MS centers. The proposed model can obtain optimum MS centers, location and link constructing or improving to minimize cost while also taking into account the expected transportation cost after failures of MS centers. The goal is to choose facility location and link constructing or improving that are both inexpensive under traditional objective functions and also reliable. In other words, the model provides an enough effective, and confidant approach to be applied in MS systems regarding the failure probability of MS centers. Clearer descriptions of the proposed problem are given at the following sections.

The rest of the paper is organized as follows. In Section 2, a brief review of the location models relevant to planning MS systems and the description of research gap are explained. In Section 3, the multiobjective mathematical model formulation of reliable facility location network design problem (RFLNDP) for optimizing MS systems is developed. In Section 4, the linearization of the proposed model is presented, and a case study that exactly shows the application of the model formulation is demonstrated and solved by the model in Section 5. Sensitivity analysis of the model parameters is reported in Section 6. Finally, conclusions and future works are presented.

\section{Literature Review and Description of Research Gap}

In order to place our contribution in the right perspective, we briefly review two main streams of the literature that may be of interest for comparison: the literature on MS location problems and on facility location problem regarding network design and also system reliability topics. Clearly these two research areas are strongly related; having the location of MS centers inspired a significant amount of research in facility location theory considering to the network design and system reliability topics.

2.1. Location Models Related to MS Systems. As reviewed by Brandeau and Chiu (1989) [4], Eiselt and Laporte (1995) [5], Owen and Daskin (1998) [6], Hale and Moberg (2003) [7], ReVelle and Eiselt (2005) [8], and Daskin (2008) [9], there have been research results on facility location problems in various areas such as distribution systems, telecommunication networks, transportation networks, and health care systems. When a set of demand locations and a set of candidate facility locations are given, the facility location problems are usually concerned with the decisions on where to locate facilities in order to minimize the total cost for constructing facilities and satisfying demands subject to a set of constraints. For instance, Lim and Kim (2001) [10], Correa et al. (2004) [11], Lorena and Senne (2004) [12], Fathali and Kakhki (2006) [13], T. Drezner and Z. Drezner (2007) [14], Osman and Ahmadi (2007) [15], Resende and Werneck (2007) [16], and Domínguez and Muñoz (2008) [17] considered problems of finding locations of the facilities with the objective of minimizing the cost, weighted travel distance, or the number of facilities. On the other hand, Galvão et al. (2000) [18], Hong and Lee (2004) [19], O. Karasakal and E. K. Karasakal (2004) [20], Weng et al. (2006) [21], Berman et al. (2007) [22], and ReVelle et al. (2008) [23] studied the problems with the objective of maximizing the number of demand locations covered by the facilities.

There have also been many research papers on facility location problems in MS systems, as reviewed by Rahman and Smith (2000) [24], Daskin and Dean (2005) [25], and D. G. Kim and Y. D. Kim (2010) [26]. One of the commonly considered location problems is related to the emergency medical service (EMS) systems. Toregas et al. (1971) [27] and Daskin and Stern (1981) [28] modeled the location problems of EMS facilities and EMS vehicles as set covering problems of finding the minimum number of facilities or EMS vehicles required to satisfy the demand for the service. On the other hand, Daskin (1997) [29] presented a planning framework to help health planners make decisions on locations of health care facilities, and Marianov and ReVelle (1996) [30] developed a probabilistic model for determining the locations of emergency service facilities. In addition, Verter and Lapierre (2002) [31] considered a problem of locating preventive health-care facilities, and Jia et al. (2007) [32] dealt with a facility location problem for a large-scale EMS system. Beraldi and Bruni (2009) modelled the general and complex problem of designing and planning emergency 
medical services as a two stage stochastic programming problem with probabilistic constraints [33] Mete and Zabinsky (2010) developed a stochastic programming approach for disaster preparedness to plan the storage and distribution of medical supplies to be used in emergencies [34]. Their models provide interdisciplinary agencies with a means to prepare and respond to disasters by efficiently balancing the risk. D. G. Kim and Y. D. Kim (2010) considered a long-term care facility location problem with the objective of balancing the loads of the facilities under the assumption that patients are served by the nearest facilities [26]. Coskun and Erol (2010) presented an optimization integer programming model to decide locations and types of service stations, regions covered by these stations under service constraints with a minimum total cost of the overall system [35]. Mestre et al. (2012) have proposed a hierarchical and multiservice model to help health care planners to decide upon the location and structure of hospital supply when their main objective is to improve the access to hospital services [1]. Yujun et al. (2012) proposed an intelligent computation framework for disaster rescue operation planning, which bridges two popular computing paradigms: agent computing and evolutionary algorithms. In the proposed framework, various kinds of EAs are encapsulated in the form of distributed, component-based agents, which cooperate with each other in asynchronous teams to produce and evolve the problem solutions [36].

\subsection{Facility Location with Respect to Network Design and Sys-} tem Reliability Topics. One important matter in the modeling of facility location problems is to propose an efficient mathematical model which can illustrate an effective description of the problem and therefore considerably reduces its related costs. Two significant topics that can help to reach such goal are network design and reliability of system. The importance of these topics in modeling of facility location problems will be explained further.

Considering simultaneously the facility location and network design, the proposed problem can be described in more realistic formulation and modeling. As we know, the classical facility location problems, including $P$ median and $P$ center problems [37], the uncapacitated facility location problems [38], the maximum covering location problems [39], and the set covering location problems [27], have been widely utilized to analyze and determine the locations of public and private facilities.

All of the aforementioned classical models locate facilities on a predetermined network. However, the topology of the underlying network may profoundly impact upon the optimal facility locations and can have many applications in industries and services. In the literature review, it is evident that Daskin et al. in 1993 introduced the first initial model of facility location-network design problem (FLNDP) [40]. They presented some preliminary results which showed the effect of network design topic in mathematical modeling of facility location problems and their optimal solution. Later, Melkote [41] in his doctoral thesis researched three models for the FLNDP including UFLNDP, the capacitated facility locationnetwork design problem (CFLNDP), and the maximum covering location-network design problem (MCLNDP). The results of the thesis were published in $[42,43]$. Drezner and Wesolowsky [44] proposed a new network design problem with potential links; each of which could be either constructed at a given cost or not. Moreover, each constructed link could be constructed as either a one-way or two-way link. They developed four basic problems subject to two objective functions: finally, they solved the problems by a descent algorithm, a simulated annealing, a tabu search, and a genetic algorithm as main solution procedures. In another doctoral thesis, Cocking $[45,46]$ expanded some efficient approaches to solve the static budget constrained (FLND) problem. Some useful algorithms were developed to find good upper bounds and good lower bounds on the optimal solution. Simple greedy heuristics, a local search heuristic, metaheuristics including simulated annealing (SA) and variable neighborhood search (VNS), as well as a custom heuristic based on the problem-specific structure of FLND were the main heuristics and metaheuristics that were proposed in Cocking's doctoral thesis. Besides, a branch-and-cut algorithm using heuristic solutions as upper bounds and cutting planes to improve the lower bound of the problem were developed. The method reduced the number of nodes which was needed to approach optimality. Recently, Bigotte et al. [47] have proposed a mixed integer optimization model for integrated urban hierarchy and transportation network planning. The model simultaneously determines which urban centers and which network links should be transferred to a new level of hierarchy in order to improve availability of all groups of facilities.

Reliability is another significant subject that can affect facility location and allocation. The importance of the reliability of system is recognized when a set of the facilities has been constructed, but one or some of the facilities occasionally become unavailable in situations such as inclement weather, labor actions, sabotage, or changes in ownership. There are different types of such catastrophic plight, many of which caused facilities to shut down including a series of mail-based anthrax attacks in the United States in 20012002 [48-51] and SARS outbreak in Toronto, Canada, in the summer of 2003 [51]. It is observed that when a facility failure occurs, customers may have to be reassigned from their original facilities to the other available facilities; a condition that surely requires higher transportation costs. Therefore, study of facility location problems with respect to the reliability of system and also network design can practically ameliorate solving of the mentioned problems in industries and services by obtaining more accurate and efficient solutions.

In the traditional locational analysis literature, Snyder and Daskin in 2003 were the first to propose an implicit formulation of the stochastic $P$ median and fixed charge problems based on level assignments, in which the candidate sites are subject to random disruptions with equal probability [48-50]. Shen et al. [52] and Berman et al. [51] relaxed the assumption of uniform failure probabilities, formulated the stochastic fixed-charged facility location problem as a nonlinear mixed integer program, and expanded several heuristic solution algorithms. Berman et al. [51] concentrated 
on an asymptotic property of the problem and verified that the solution to the stochastic $P$ median problem coincides with the deterministic problem as the failure probabilities approach zero. They also presented some efficient heuristics with bounds on the worst-case performance. Lim et al. [53] suggested a reliability continuum approximation (CA) approach for facility location problems with uniform customer density. For simplification, a specific form of failureproof facility was supposed to exist; a customer was always reassigned to a failure-proof facility after its nearest regular facility failed, regardless of other regular facilities. With respect to the huge investment for facility location and network design, the attention to the failures of system based on facility disruptions in facility locating and network design has been increased recently [54-56].

Peng et al. [57] studied the effect of considering reliability topic on logistic networks design with facility disruptions and illustrated that applying a reliable network design is often possible with negligible increases in total location and allocation costs depending on decision makers' opinion. They considered the commodity production/delivery system without respect to open/close decisions on the arcs of supply chain system and, by applying the $p$ robustness criterion (which bounds the cost in disruption scenarios), they simultaneously minimize the nominal cost (the cost when no disruptions occur) and reduce the disruption risk. Recently, Jabbarzadeh et al. [58] studied a supply chain design problem with the risk of disruptions at facilities and formulated the problem as a mixed integer nonlinear program which maximizes the total profit for the whole system. The proposed model simultaneously determines the number and locations of facilities, the subset of customers to serve, the assignment of customers to facilities, and the cycle-order quantities at facilities.

2.3. Description of Research Gap. Although the literature on MS center location problems is abundant, there are not any studies in which MS center location problems were considered regarding link network design and system reliability topics. Also, as it is obvious from the related literature, study of facility location problems with considering network design and reliability of system is relatively rare. Moreover, the existing studies have not considered both network design and reliability of system altogether on facility location, while, with considering only one of them, they have proposed different solving methods such as exact, heuristics, and metaheuristics. Also, the literature review of this study shows that there is an unfulfilled research in facility locating with respect to more realistic factors such as network design and reliability of system to manage the practical facility location problems. However, there are numerous practical instances of facility location problems in which simultaneous consideration of network design and reliability of system can lead to a more realistic and practical mathematical modeling of the problem. Locating of MS centers can be introduced as the most obvious and practical example. As a result, proposing a new mathematical model formulation, which can obtain optimum facility location and link constructing under some special conditions, such as reliability of system, can lead decision makers to more accurate solutions for the considered problem. In other words, the proposed model provides an enough effective and confidant approach to be applied by different decision makers especially health care planners in locating several facilities for improving the efficiency and responsibility of them.

In this paper, we develop a new integrated approach to facility location problems with respect to the subjects of network design and system reliability as it can be named reliable facility location network design problem (RFLNDP) for improving the efficiency of MS systems. In our approach, the goal is to determine the following:

(i) the optimum locations of new MS centers regarding network design and system reliability,

(ii) the primary MS center and backup MS center of every demand node (population center),

(iii) the transportation links that should be constructed or improved in the proposed network,

(iv) the amount of demands of nodes that should be transported by the transportation links,

(v) the fraction of every demand that should be supplied by new and exciting MS centers.

The motivation of this research is to consider simultaneously two practical factors (network design and reliability of system) to develop the mathematical modeling of MS center location problems, which has not been considered until now based on the authors' best knowledge. The main contributions that differentiate this paper from the existing ones in the related literature can be summarized as follows.

(i) Introducing a new multiobjective mathematical optimization model to consider simultaneously MS center location and allocation, network design, and reliability of systems as a mixed integer, nonlinear program (MINLP) problem. A model that integrates the managerial and strategic decision making such as determining the optimum locating of new MS centers, optimum constructing or improving of the transportation links, and optimum allocating demand nodes to located MS centers so that locating, allocating, link constructing or improving, and transporting costs as well as reliability of systems are optimized.

(ii) Our new mathematical formulation not only takes into account the MS center location costs, link construction or improvement costs, and transportation costs, but also considers the reliability of MS centers as the failure costs of MS centers in an MS system.

(iii) Specifically, we consider and explain the RFLNDP in a practical case study which exactly shows the application of new proposed mathematical model in improving the efficiency of an MS system at a province. 


\section{Mathematical Model Descriptions}

3.1. Definition. In this section, the general structure of the mentioned problem is exactly described. Suppose that a set of towns as demand nodes exists in a geographical region and a set of roads as transportation links that contains existing and new candidate links is defined to construct a transportation network on the mentioned region. A set of MS centers (e.g., clinics) exists in the region, and it is clearly desired to locate a set of new MS centers, to construct new candidate links, and to improve existing links so that the total investment costs (including locating facilities, constructing links, and improving existing link) and total operational costs (including transportation costs) are minimized. One point that should be considered is that all of the mentioned MS centers (containing existing and new facilities) are not reliable and due to some unexpected events, such as inclement weather, labor actions, lack or shortage of doctor or drug, sabotage, or changes in ownership, they occasionally fail and become unavailable. Accordingly, the demand nodes of failed MS centers must be assigned to the nearest active MS center. Therefore, increasing the traveled distances by demand nodes raise transportation costs. If the increase in transportation costs is considered as failure cost [21,22], then an objective function can be defined as "failure cost" in which failure costs of MS centers can be considered and optimized.

The problem is to determine (1) the optimum locations of new MS centers regarding network design and reliability of system, (2) the primary MS center and backup MS center of every demand node, (3) the transportation links that should be constructed or improved in the proposed network, (4) the amount of demands of nodes that should be transported by the transportation links, and (5) the fraction of every demand that should be supplied by new and exciting MS centers.

Assumptions. The assumptions for RFLNDP can be described as follow.

(1) Each node of network shows a town or urban residence center as a demand point.

(2) The MS centers and network links (transportation roads) are uncapacitated.

(3) New MS centers can only be located on the nodes (towns) of the network and may not be located on the links of network.

(4) At most, one new MS center can be located on each node.

(5) The general structure of the network is planned based on a customer-to-server system, which means that the demands themselves travel to the relevant MS centers in order to be served.

(6) All travel costs are symmetric.

(7) All network links are directed.

(8) Locating new MS centers and allocating demand nodes are considered so that the MS center location costs, link construction or improvement costs, and transportation costs (as the first objective function) as well as failure costs of system (as the second objective function) are simultaneously optimized, subject to if any MS center fails, then, by reassigning the demand nodes to the available MS centers, the resulting costs, are known as the MS center failure costs and the second objective function.

(9) It is may happen that several MS centers simultaneously fail and are not be available at a time.

(10) Based on the geographical situation, the existing and new MS centers can be divided in two categories: the nonfailable MS centers and the failable MS centers. In fact, the failable or nonfailable property of MS centers depend on the type of geographic area of demand node that MS center is located on it.

(11) In order to simplify the calculation of the total costs and control the complexity of the problem, neither the probability nor the duration of a failure will be considered. In fact, our goal is simply to hamper the cost that results from a failure, regardless of how frequently this cost incurs.

\section{Notifications}

Parameters

$P$ : number of new MS center to open, $(P \geq 2)$

$F$ : set of failable nodes in the network

$N F$ : set of nonfailable nodes in the network

$N$ : set of nodes in the network $(N F \cup F=$ $N \& N F \cap F=\varnothing)$

$M$ : set of links in the network (including existing and new candidate links)

$\alpha$ : weight on objectives $(0 \leq \alpha \leq 1)$

$q$ : probability that a failable MS center will fail $(0 \leq q \leq 1)$

$\mathrm{BC}$ : upper limit of investment budget constraint

$d_{i}$ : demand at node $i \in N$

$f_{i}$ : fixed cost of locating a MS center at node $i \in N$

$c_{i j}$ : cost of constructing or improving link $(i, j)$

$t_{i j}^{0}$ : transportation cost of a unit flow on link $(i, j)$

$t_{i j}^{l}$ : transportation cost of a unit flow of demand node $l$ on link $(i, j)=t_{i j}^{0} d_{l}$.

We assume all parameters are integer valued except all kinds of costs. As an important point, it is mentioned that $t_{i j}^{0}\left(t_{i j}^{l}\right)$ presents a link-specific transportation cost, not an origin-destination transportation cost, and we have to utilize link-specific transportation cost as an initial parameter of RFLNDP model because in RFLNDP, unlike RFLP, the network is not known in advance. Hence, we cannot calculate origin-destination transportation costs. 
Variables:

$Z_{i k r}:\left\{\begin{array}{r}1, \quad \begin{array}{l}\text { If a MS center is located at node } i \text { as a } \\ \text { primary MS center and the MS center } \\ \text { located at node } k \text { is the } i \text { 's back up }\end{array} \\ \text { MS center at level } r \\ 0, \quad \text { Otherwise, }\end{array}\right.$

$X_{i j}: \begin{cases}1, & \text { If link }(i, j) \text { is constructed or improved } \\ 0, & \text { Otherwise }\end{cases}$

$S_{i}: \begin{cases}1, & \text { If a MS center is located at node } i \\ 0, & \text { Otherwise }\end{cases}$

$Y_{i j}^{l}$ : demands of node $l$ on link $(i, j) \in M$

$Y_{i j}^{i}=X_{i j}(i, j) \in M$

$W_{i}^{l}$ : demands of node $l$ served

by a MS center at node $i \in N$

$W_{i}^{i}: S_{i} i \in N$.

3.2. Model Formulation. Using these notations and assumptions, the two multiobjectives of RFLNDP can be defined as follows:

$$
\begin{aligned}
& w_{1}=\sum_{(i, j) \in M} t_{i j}^{i} X_{i j}+\sum_{(i, j) \in M} \sum_{l \in N: l \neq i} t_{i j}^{l} Y_{i j}^{l}, \\
& w_{2}=\sum_{i \in N}\left[\sum_{k \in N F} \sum_{r=1}^{P-1} q^{r} Z_{i k r}\right. \\
& \times\left[\sum_{j \in N} \sum_{l \in N: l \neq j} t_{j k}^{l} Y_{j i}^{l}+\sum_{j \in N} c_{j k} X_{j i}\right. \\
& \left.+\sum_{i \in N}\left[\sum_{k \in F} \sum_{r=1}^{P-1} q^{r}(1-q) Z_{i k r} t_{j k}^{0} d_{j} X_{j i}+c_{i k}+t_{i k}^{i}\right]\right] \\
& \times\left[\sum_{j \in N} \sum_{l \in N: l \neq j} t_{j k}^{l} Y_{j i}^{l}+\sum_{j \in N} c_{j k} X_{j i}\right. \\
& \left.\left.+\sum_{j \in N} t_{j k}^{0} d_{j} X_{j i}+c_{i k}+t_{i k}^{i}\right]\right]
\end{aligned}
$$

Objective $w_{1}$ computes the transportation costs among different demand nodes and existing and new MS centers. Also, objective $w_{2}$ calculates the expected failure costs so that each demand of node $j$, served by MS center $i$, is served by its level- $r$ MS center (call it $k$ ) if the $r$ closer MS centers have failed (this occurs with probability $q^{r}$ ) and $k$ itself has not failed (this occurs with probability $1-q$ if $k \in F$ ) and (with probability 1 if $k \in N F$ ). Different approaches have been proposed to solve the multiobjective decision problems. Regarding the structure of the proposed problem, we consider the weighting method of multiobjective programming, and our model minimizes a weighted sum $\alpha w_{1}+(1-\alpha) w_{2}$ of the two objectives, where $0 \leq \alpha \leq 1$. By solving the problem for various values of $\alpha$, one can generate a trade-off curve between the transportation costs and expected failure cost using the weighting method of multi-objective programming. So, the mathematical formulation of the RFLNDP is shown below:

S.T.

$$
\begin{aligned}
& \text { Model (I) } \sum_{i \in N} \sum_{k \in N} f_{i} Z_{i k} \\
& +\sum_{(i, j) \in M} c_{i j} X_{i j} \leq B C, \\
& S_{i}+\sum_{j \in N} X_{i j}=1 \quad \forall i \in N, \\
& X_{l i}+\sum_{j \in N: j \neq l} Y_{j i}^{l}=\sum_{j \in N} Y_{i j}^{l}+W_{i}^{l}, \\
& \forall i, l \in N: i \neq l, \quad \forall(l, i) \in M, \\
& \sum_{j \in N: j \neq l} Y_{j i}^{l}=\sum_{j \in N} Y_{i j}^{l}+W_{i}^{l} \\
& \forall i, l \in N: i \neq l, \quad \forall(l, i) \notin M, \\
& S_{l}+\sum_{i \in N: i \neq l} W_{i}^{l}=1 \quad \forall l \in N, \\
& Y_{i j}^{l} \leq X_{i j} \quad \forall(i, j) \in M, \forall l \in N: i \neq l, \\
& W_{i}^{l} \leq S_{i} \quad \forall i, l \in N: i \neq l, \\
& X_{i j}+X_{j i} \leq 1 \quad \forall(i, j) \in M \text {, } \\
& \sum_{i \in N} S_{i}=P, \\
& \sum_{r=1}^{p} Z_{i k r} \leq 1 \quad \forall i \in N, \forall k \in N, \\
& S_{i} \geq Z_{i k r} \quad \forall i \in N, \forall k \in N, \\
& r=1,2, \ldots, P-1, \\
& \sum_{k \in N} Z_{i k r}+\sum_{k \in N F} \sum_{s=1}^{r-1} Z_{i k s}=1 \quad \forall i \in F F, \\
& r=1,2, \ldots, P-1,
\end{aligned}
$$




$$
\begin{gathered}
Z_{i k r} \leq \sum_{m \in N} Z_{k m r} \quad \forall i \in N, \forall k \in N, \\
r=1,2, \ldots, P-1, \\
Y_{i j}^{l} \geq 0 \quad \forall i, j, l \in N, \\
W_{i}^{l} \geq 0 \quad \forall i, l \in N, \\
S_{i} \in\{0,1\} \quad \forall i \in N, \\
X_{i j} \in\{0,1\} \quad \forall(i, j) \in M, \quad \forall l \in N: l \neq i, \\
Z_{i k} \in\{0,1\} \quad \forall i, k \in N: l \neq i .
\end{gathered}
$$

The objective function (3) illustrates the weighting sum of two objective functions. Constraint (4) shows the investment budget constraint and investigates how we can have best allocation of limited investment budget to the construction of new MS centers and link roads or improvement of roads. In general observation, Constraints (5)-(8) consider the rational conditions of the transportation flow between demand nodes and MS centers. Specifically, Constraint (5) ensures that demand at $i$ is either served by an MS center at $i$ or by shipping on some link out of $i$. Constraints (6) and (7) state conservation of flow for transshipped demand. Constraints (8) impose that the demand of node $l$ must find a destination, whether it is estimated by node $l$ itself $\left(z_{l k}\right)$ or by the other nodes $i\left(W_{i}^{l}\right)$. Constraints (9) and (10) guarantee that potential links and MS centers are not used if they are not constructed. Constraints (13) emphasize that, on any given link, an optimal solution flow will be in only one direction. Therefore, both links $(i, j)$ and $(j, i)$ cannot be constructed or improved simultaneously. Constraint (12) restricts the total number of newly located MS centers to the predetermined MS centers of $P$. Constraints (13) represent that every MS center can be selected as a backup of a primary MS center at most on one level. Constraints (14) guarantee that allocation of MS centers in different levels of reliability is not done if an MS center is not located or constructed. Constraints (17) illustrate that for each primary MS center $i$ at level $r$, a MS center either is assigned as a backup of $i$ at level- $r$ or is assigned to a backup non-failable MS center at level-s $(s<r)$. Constraints (16) prohibit assignments to a MS center that has not been opened. Constraints (17)-(18) force the flow variables to be non-negative, while Constraints (19)-(21) enforce the binary restriction on the primary and backup MS center location and link decision variables.

As mentioned, according to the single assignment property, every demand of node is completely assigned to the closest single MS center. That is, nothing is gained by "splitting up" a demand and sending parts of it to different MS centers. Therefore, the fractions of demands, which served a single MS center, are integer-valued, while $W_{i}^{l}$ and $Y_{i j}^{l}$ are integral [6].

\section{Linearization of Mathematical Model}

The mathematical model (I) of RFLNDP is a mixed integer nonlinear programming (MINLP) model because the proposed model has non-linear terms in the second objective function $\left(w_{2}\right)$. However, it can be easily linearized by introducing new binary variables and additional constraints as follows:

$$
U_{j i k r}=Z_{i k r} \times X_{j i}, \quad V_{i j k r}^{l}=Z_{i k r} \times Y_{j i}^{l},
$$

and by introducing the following constraints:

$$
\begin{aligned}
& U_{i j k r} \leq Z_{i k r} \quad \forall i, j, k \in N, r=1,2, \ldots, P-1, \\
& U_{i j k r} \leq X_{j i} \quad \forall i, j, k \in N, r=1,2, \ldots, P-1, \\
& U_{j i k r} \geq Z_{i k r}+X_{j i}-1 \quad \forall i, j, k \in N, r=1,2, \ldots, P-1, \\
& V_{i j k r}^{l} \leq M Z_{i k r} \quad \forall i, j, k, l \in N, r=1,2, \ldots, P-1, \\
& V_{i j k r}^{l} \leq Y_{j i}^{l} \quad \forall i, j, k, l \in N, r=1,2, \ldots, P-1 \\
& V_{i j k r}^{l} \geq Y_{j i}^{l}-M\left(1-Z_{i k r}\right) \quad \forall i, j, k, l \in N, \\
& r=1,2, \ldots, P-1 .
\end{aligned}
$$

Consequently, objective function $\left(w_{2}\right)$ can be substituted with the following:

$$
\begin{aligned}
& w_{2}=\sum_{i \in N}\left[\sum_{k \in N F} \sum_{r=1}^{P} q^{r}\right. \\
& \times\left[\sum_{j \in N} \sum_{l \in N: l \neq j} t_{j k}^{l} V_{i j k r}^{l}+\sum_{j \in N} c_{j k} U_{i j k r}\right. \\
& \left.\left.+\sum_{j \in N} t_{j k}^{0} d_{j} U_{i j k r}+c_{i k} Z_{i k r}+t_{i k}^{i} Z_{i k r}\right]\right] \\
& +\sum_{i \in N}\left[\sum_{k \in F} \sum_{r=1}^{P} q^{r}(1-q)\right. \\
& \times\left[\sum_{j \in N} \sum_{l \in N: l \neq j} t_{j k}^{l} V_{i j k r}^{l}+\sum_{j \in N} c_{j k} U_{i j k r}\right. \\
& \left.\left.+\sum_{j \in N} t_{j k}^{0} d_{j} U_{i j k r}+c_{i k} Z_{i k r}+t_{i k}^{i} Z_{i k r}\right]\right] .
\end{aligned}
$$

Therefore, the final model (I) of RFLNDP converted to MILP easily.

\section{Describing an Application of the Proposed Model by A Case Study}

The application of the mathematical model (I) is described as a practical case study, the goal of which is to improve accessibility to MS centers for the urban residence centers (towns) in a province of Iran named Khorasane Shomali. 


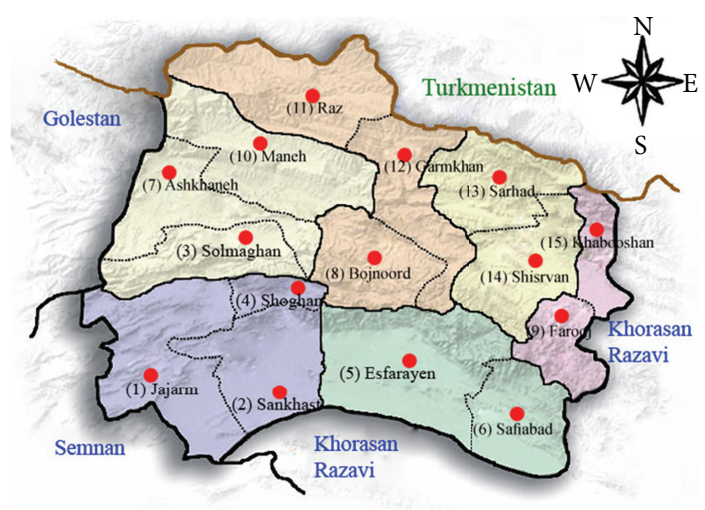

FIGURE 1: Geographical map of Khorasane Shomali province with urban residence centers.

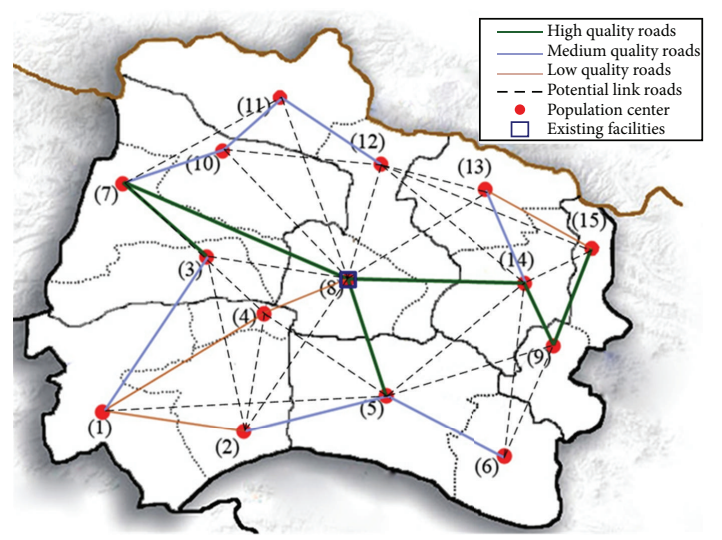

FIGURE 2: The map of different roads of Khorasane Shomali province.

Khorasane Shomali, with $28434 \mathrm{~km}^{2}$ of area, is known as one of the deprived areas in the country that requires a special attention in order to move toward a comprehensive and sustainable development. In addition, with respect to its geographical position and its adjacency with Turkmenistan country, the importance of this special attention has been increased. One of the crucial indicators in efficient development of a province or area is health care and medical system conditions that have a considerable effect on promotion of province development indicators and also improvement of public health welfare. In this study, the aim is to consider the health care and medical system of the Khorasane Shomali so that regarding the limited allocated budget to promote the medical system of the province, the best and most efficient design and planning is provided in order to improve the medical system conditions.

As it can be seen on the map in the Figure 1, Khorasane Shomali consists of 15 urban residence centers or towns (nodes) with total population of 720809 .

Reliable data were collected, as far as possible, for the problem. There is just one available MS center as a clinic that is located in the center of the province (Bojnourd) that, with respect to the geographical situation and other technical reasons, this clinic is known as a reliable MS center. Also other residence centers $(15-1=14$ residence centers that do not have any health care service centers) are known as potential nodes to open new MS centers (new facilities).

According to the current conditions, roads in Khorasane Shomali province are classified into three categories in term of quality: high, medium, and low. In fact, depending on the type of the roads, constructing or improving costs vary; as a result, low and medium quality roads can be upgraded to high quality roads with lower constructing costs.

Figure 2 shows the residence centers and the road network of Khorasane Shomali province, as well as the existing MS centers in cities of the province. It can be seen that there are 17 existing and 23 potential links or roads which have three different qualities and picture with various thicknesses in the graph of Figure 2.

The transportation cost for each client in kilometer is randomly calculated subject to a discrete uniform distribution in $(0.10,0.15)$. The construction cost of new roads and improvement cost of the existing roads are calculated per kilometer and are between (100000 and 400000) as many of transportation cost according to their qualities. Each residence center is a client node with a demand that equals its population. The fixed cost of opening an MS center depends on the fact that various urban status of towns varies between (1913880.60 and 6394276.00).

In addition, because of some reasons including bad weather conditions, delay in drug supply, lack of specialists or service personnel, staff strikes, and the occurrence of natural disasters such as floods and earthquakes, it is possible that each MS center cannot serve its customers; a condition that provokes an MS center to fail. Since such condition is true for all centers in the province, all of them are unreliable (the center of province (i.e., Bojnoord) is exception and is known as reliable MS center at all mentioned conditions because of significant development of it). Based on the geographical, natural, and other important conditions in Khorasane Shomali province, it is desired that two objective functions including transportation costs and failure costs are defined so that not only the transportation costs are reduced but also the long-run expected costs due to failures are minimized (i.e., maximize the system reliability). Other complementary information contains distances among different cities, transportation cost per unit flow, construction cost of new roads, and improvement cost of existing roads; the rest of complementary information which is about different cities of Khorasane Shomali province has been mentioned in the Appendix, respectively.

It is worth mentioning that Ministry of Health and Medical Education and Ministry of Road and Transportation are responsible for investment in MS centers and road network construction or improvement, respectively, and they should provide a comprehensive plan to improve the quality of health services in each province. According to the medical equipment limitations and other technical reasons, the ministry of health and medical education has also determined that just two new health care centers can be located in Khorasane Shomali and, because of one existing health care center, the value of $P$ is 3 (as a reminder point, the facility location cost $\left(f_{i}\right)$ of existing health care center is zero, i.e., $\left.f_{8}=0\right)$. 


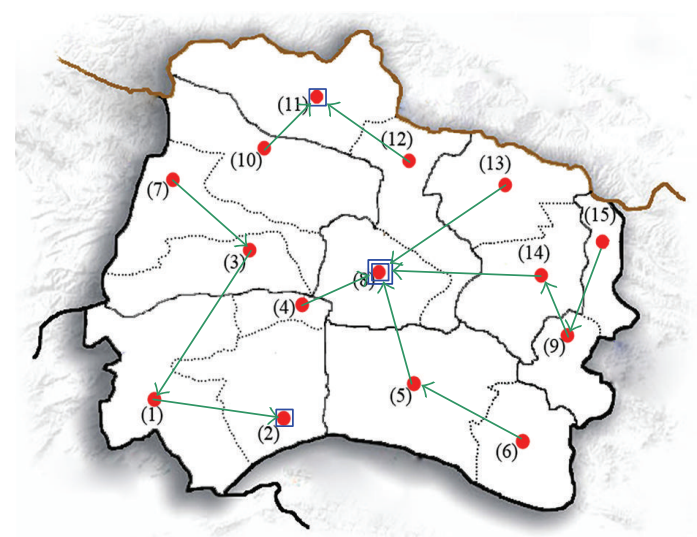

FIgURE 3: The optimal solution of the case study.

In order to improve the physical access to the MS centers in Khorasane Shomali, the ministries have investment constraint and the budget constraint is determined $30000000 \mathrm{MU}$ (Monetary Unit). The main goals in the considered case study are to determine the following:

(i) the optimum locations of new MS centers based on the two mentioned objective functions,

(ii) the primary MS center and backup MS centers of every residence center for each reliability level,

(iii) the transportation links that should be constructed or improved in the proposed network,

(iv) the amount of demands of residence centers that should be transported by transportation links,

(v) the fraction of every demand that should be supplied by new and exciting MS centers.

According to the mentioned conditions, it is evident that the case study can be exactly investigated as a reliability facility location-network design problem (RFLNDP). As a result, according to the mentioned description, the Model (I) is a suitable mathematical modeling for the case study. Therefore, as a propositional option to decrease transportation costs and also the long run expected costs due to failures, the mathematical model (I) can suggest that new MS centers can be established in the nodes in which no MS center has been located. Also, constructing new roads or improving existing roads as it is shown in Figure 3 can be suggested as the other propositional option to improve the two mentioned objective functions of the case study.

Based on the urban specifications of Khorasane Shomali province, the values of $q$ and $\alpha$ are 0.15 and 0.85 , respectively. Also, it is desired that just two new MS centers are located $(P=2)$. Due to the above description and the predetermined value of different parameters, the case study was modeled by the model (I) and coded in GAMS and solved by CPLEX solver. The results are presented in Figure 3 which visually illustrates the obtained optimal solution. As Figure 3 shows, the values of $S_{10}$ and $S_{14}$ are determined to 1 . This means that the optimum locations for two new MS centers are residence centers 10 and 14. Also, with respect to the several costs (i.e., transportation costs, link construction or improvement cost, and also MS center location costs); the MS center 8 is determined as backup MS center of MS center 10 in reliability level 2, and 3, respectively. Also, the MS center 8 is determined as backup MS center of MS center 14 in reliability level 2 and 3 , respectively. It is mentioned that in our case study, the nonfailable MS center 8 is determined as the backup MS center of the specified primary MS centers in different reliability levels, but some case studies can be raised that different failable MS centers is determined for different reliability levels.

In addition, just three new roads should be constructed between node 3 and 8 , nodes 12 and 8 , and also nodes 6 and $9\left(X_{3,8}=1 ; X_{12,8}=1 ; X_{6,9}=1\right)$. Also, the quality of roads between nodes 2 and 5 , nodes 7 and 10 , nodes 11 and 10 , and nodes 13 and 14 should be improved from medium to high. $\left(X_{3,1}=1 ; X_{6,5}=1 ; X_{12,11}=1, X_{10,11}=1\right)$. Also the quality of roads between nodes 1 and 4 and nodes 4 and 8 should be improved from low to high. $\left(X_{2,1}=1 ; X_{4,8}=1\right)$.

As Figure 3 presents, the population of some cities should be transferred directly to the MS center located in the identified city. But the population of other cities should be transferred to the MS center located in the determined city via some intermediate cities. The optimal value of objective function is $6432998.614 \mathrm{MU}$ that, in detail, the value of the first objective function (the transportation costs) approximately is $6160527.400 \mathrm{MU}$, the value of the second objective function (the costs of failures) approximately is 7977002.160 MU.

As a remarkable point, the CPU time of the model is one of the key factors at applying the proposed methodology. The problem sets from 3 to 20 urban residence centers are generated randomly and solved on a PC with Intel Core 2 Duo $3.00 \mathrm{GHz}$ microprocessor and $3 \mathrm{~GB}$ RAM. Figure 4 presents the changing procedure of $\mathrm{CPU}$ time according to different increasing values in the number of urban residence centers.

As expected, the CPU times increase exponentially. Therefore, the model is not suitable for problems more than 20 urban residence centers. However, an overall glance at different provinces (especially deprived provinces) in most of countries in all of the world shows that the proposed methodology can be applied efficiently for many of different provinces of the world and a problem with 20 urban residence centers is large enough for most (deprived) provinces in different countries.

\section{Sensitivity Analysis}

In order to consider various parameters in the proposed mathematical model and the optimal solution, a relatively thorough sensitivity analysis has been conducted based on different variations in the range of the parameters of the model (I). Several sensitivity analyses can be arranged based on different parameters but a selected sensitivity analysis which is carried out in three different subsections is described as follows.

6.1. Changes of the Probability of Failing of MS Center (q) and the Weight of Objectives ( $\alpha$ ). The probability of failing of MS center $(q)$ and the weight of objective functions $(\alpha)$ 


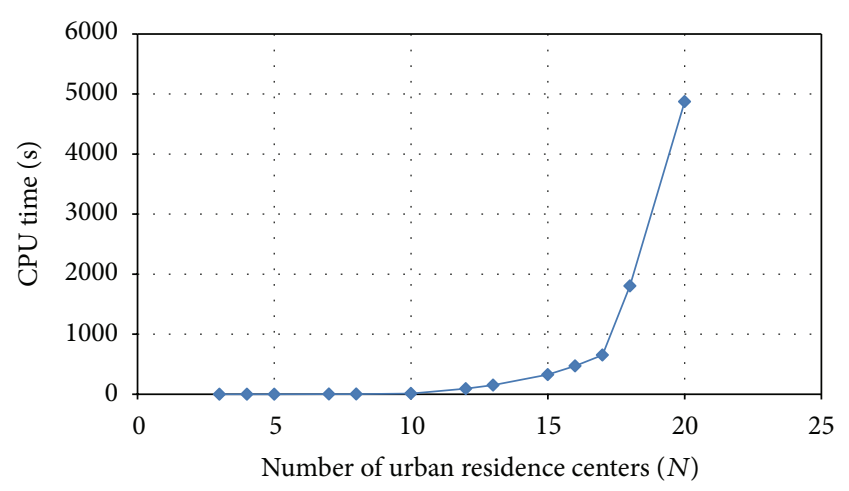

FIGURE 4: The changing procedure of CPU time for different sizes of problem.

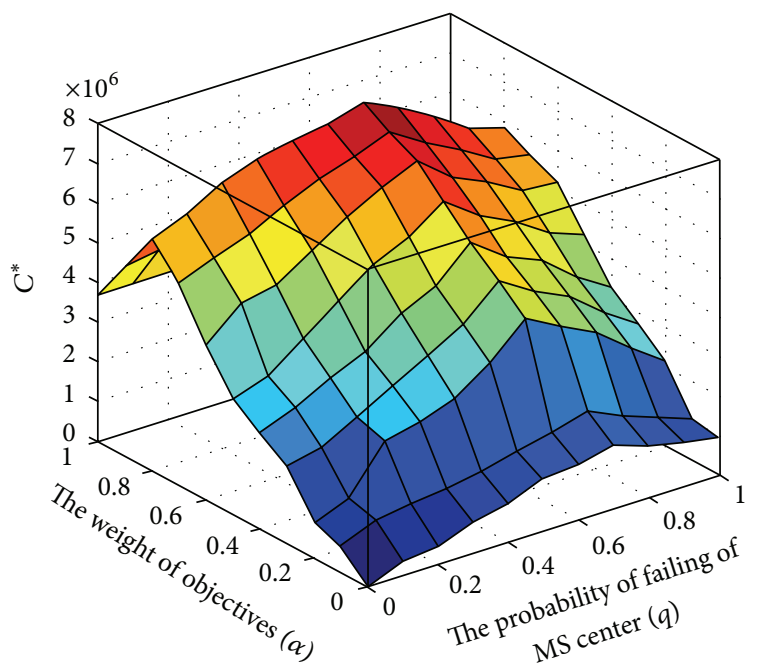

FIGURE 5: The changing procedure of $C^{*}$ according to the probability of failing of MS center $(q)$ and the weight of objectives $(\alpha)$.

are two significant parameters of the model (I) that can affect the optimal solution. Figure 5 shows the changing procedure of $C^{*}$ according to the changes in values of two mentioned significant parameters. According to Figure 5, one can conclude that the value of $C^{*}$ will maximize for the value of approximately 0.7 for both $q$ and $\alpha$ but the value of $C^{*}$ will decrease by moving away from the mentioned summit point.

6.2. Changes of the Number of New MS Centers (P) and Investment Budget Constraint (BC). Another considerable parameters of the model (I) are the number of new MS centers $(P)$ and investment budget constraint (BC) that can have significant effect on the obtained optimal solution. Figure 6 presents the changing procedure of $C^{*}$ according to different values of the $(P)$ and $(\mathrm{BC})$. Figure 6 shows that the $C^{*}$ increases when the investment budget constraint decreases, and also for a predetermined value of the investment budget constraint, the value of $C^{*}$ will decrease; that is, the increase of $(P)$ will cause decrease in $C^{*}$.

As a general concluding remarks on sensitivity analysis of parameters in the mathematical model (I), the changes

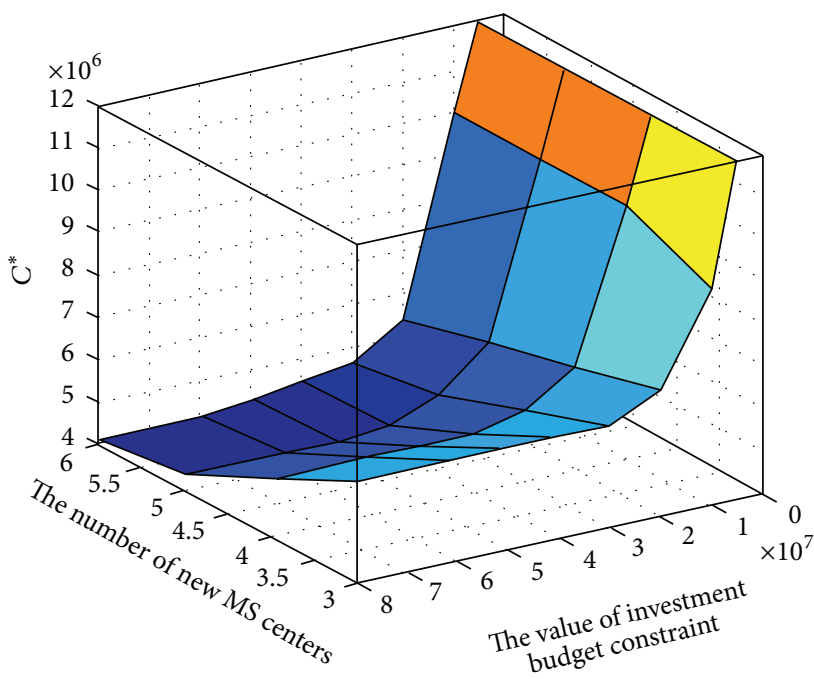

FIGURE 6: The changing procedure of $C^{*}$ for different values of new MS centers $(P)$ and investment budget constraint (BC).

of the probability of failing of MS center $(q)$ and the weight of objectives $(\alpha)$ has a significant effect on the changing procedure of the value of $C^{*}$. Moreover, the increase of the number of new MS centers $(P)$ and also the increase in investment budget constraint (BC) will caused decrease in $C^{*}$.

\section{Conclusions and Future Research}

With respect to the crucial role of efficient designing of MS systems in improving the performance and efficiency of medical services, in this paper, for the first time, a multi objective mixed integer nonlinear programming model is proposed in order to both minimize the total transportation cost of the overall system and minimize the total failure cost (maximize the reliability) of medical service centers under unforeseen situations. The proposed multi objective model determines the optimal locations of new medical system centers, optimal constructing or improving of link roads, and also optimal allocating of urban residence centers to medical service centers regarding existed, improved, or constructed link roads. Moreover, the practical case study on Khorasane Shomali province illustrates the valuable efficiency of proposed model in improving the performance of MS systems. Also, the sensitivity analysis shows an overall view of the behavior of the proposed model in response to changes of key parameters of the problem.

Our findings raise some appropriate questions for future research. First, the size of the case study is small or medium and if the size of the problem increases, a suitable solution procedure should be proposed to obtain optimal or near optimal solution. We are particularly interested in seeking apposite and efficient heuristics and metaheuristics such as tabu search (TS) and particle swarm optimization (PSO) for the mentioned propose. Second, only the RFLNDP with uncapacitated MS centers was studied in this paper; however, considering the RFLNDP as a multi objective problem 
such as minimizing the operating costs and maximizing the reliability of system with capacitated MS centers can have more practical application in MS systems. Third, one can consider more real practical factors, such as power laws, more precisely, fractals, and transportation traffic, in proposing the mathematical modeling in order to approach a more real practical model. Finally, we would explore other applications of the proposed model, especially in the fields of integrated facility sitting and network design at several MS systems. Other factors, such as power laws, more precisely, fractals, [59], transportation networking [60] and city size distribution pattern [61], data in biomedical engineering, and medicine engineering obey power laws and fractals [62-67] play a critical role in proposing more practical modeling and they can be considered in mathematical modeling. Also, working on improving the performance of medical service systems, one can consider data transmission of medical signals. However, as medical signals are fractal as can be seen from [62-67], transmitting such a type of signals may not be easy especially if they are of short memory, see details in [68]. That is also for traffic signals that are in nature fractals $[69,70]$. Finally, while discussing the interesting method for improving medical service systems and roads in transportation, one can desire to know that transportation traffic will cause air pollutions [71], which may be against the aim of medical service systems.

\section{References}

[1] A. M. Mestre, M. D. Oliveira, and A. Barbosa-Póvoa, "Organizing hospitals into networks: a hierarchical and multiservice model to define location, supply and referrals in planned hospital systems," OR Spectrum, vol. 34, no. 2, pp. 319-348, 2012.

[2] J. Current, H. Min, and D. Schilling, "Multiobjective analysis of facility location decisions," European Journal of Operational Research, vol. 49, no. 3, pp. 295-307, 1990.

[3] P. Beraldi and M. E. Bruni, "A probabilistic model applied to emergency service vehicle location," European Journal of Operational Research, vol. 196, no. 1, pp. 323-331, 2009.

[4] M. L. Brandeau and S. S. Chiu, "An overview of representative problems in location research," Management Science, vol. 35, no. 6, pp. 645-674, 1989.

[5] H. A. Eiselt and G. Laporte, "Objectives in location problems," in Facility Location: A Survey of Applications and Methods, Z. Drezner, Ed., pp. 151-179, Springer, New York, NY, USA, 1995.

[6] S. H. Owen and M. S. Daskin, "Strategic facility location: a review," European Journal of Operational Research, vol. 111, no. 3, pp. 423-447, 1998.

[7] T. S. Hale and C. R. Moberg, "Location science research: a review," Annals of Operations Research, vol. 123, no. 1-4, pp. 2135, 2003.

[8] C. S. ReVelle and H. A. Eiselt, "Location analysis: a synthesis and survey," European Journal of Operational Research, vol. 165, no. 1, pp. 1-19, 2005.

[9] M. S. Daskin, "What you should know about location modeling," Naval Research Logistics, vol. 55, no. 4, pp. 283-294, 2008.

[10] S. K. Lim and Y. D. Kim, "Plant location and procurement planning in knockdown production systems," Journal of the Operational Research Society, vol. 52, no. 3, pp. 271-282, 2001.
[11] E. S. Correa, M. T. A. Steiner, A. A. Freitas, and C. Carnieri, "A genetic algorithm for solving a capacitated p-median problem," Numerical Algorithms, vol. 35, no. 2-4, pp. 373-388, 2004.

[12] L. A. N. Lorena and E. L. F. Senne, "A column generation approach to capacitated p-median problems," Computers \& Operations Research, vol. 31, no. 6, pp. 863-876, 2004.

[13] J. Fathali and H. T. Kakhki, "Solving the p-median problem with pos/neg weights by variable neighborhood search and some results for special cases," European Journal of Operational Research, vol. 170, no. 2, pp. 440-462, 2006.

[14] T. Drezner and Z. Drezner, "The gravity p-median model," European Journal of Operational Research, vol. 179, no. 3, pp. 1239-1251, 2007.

[15] I. H. Osman and S. Ahmadi, "Guided construction search metaheuristics for the capacitated p-median problem with single source constraint," Journal of the Operational Research Society, vol. 58, no. 1, pp. 100-114, 2007.

[16] M. G. C. Resende and R. F. Werneck, "A fast swap-based local search procedure for location problems," Annals of Operations Research, vol. 150, no. 1, pp. 205-230, 2007.

[17] E. Domínguez and J. Muñoz, "A neural model for the p-median problem," Computers \& Operations Research, vol. 35, no. 2, pp. 404-416, 2008.

[18] R. D. Galvão, L. Gonzalo Acosta Espejo, and B. Boffey, "A comparison of Lagrangean and surrogate relaxations for the maximal covering location problem," European Journal of Operational Research, vol. 124, no. 2, pp. 377-389, 2000.

[19] S. H. Hong and Y. H. Lee, "The maximal covering location problem with cost restrictions," Journal of the Korean Institute of Industrial Engineers, vol. 30, pp. 93-106, 2004.

[20] O. Karasakal and E. K. Karasakal, "A maximal covering location model in the presence of partial coverage," Computers \& Operations Research, vol. 31, no. 9, pp. 1515-1526, 2004.

[21] K. Weng, C. Yang, and Y. Ma, "Two artificial intelligence heuristics in solving multiple allocation hub maximal covering problem," in Intelligent Computing, vol. 4113 of Lecture Notes in Computer Science, pp. 737-744, 2006.

[22] O. Berman, V. Verter, and B. Y. Kara, "Designing emergency response networks for hazardous materials transportation," Computers \& Operations Research, vol. 34, no. 5, pp. 1374-1388, 2007.

[23] C. ReVelle, M. Scholssberg, and J. Williams, "Solving the maximal covering location problem with heuristic concentration," Computers \& Operations Research, vol. 35, no. 2, pp. 427-435, 2008.

[24] S. Rahman and D. K. Smith, "Use of location-allocation models in health service development planning in developing nations," European Journal of Operational Research, vol. 123, no. 3, pp. 437-452, 2000.

[25] M. S. Daskin and L. K. Dean, "Location of health care facilities," in Operations Research and Health Care, pp. 43-76, 2005.

[26] D. G. Kim and Y. D. Kim, "A branch and bound algorithm for determining locations of long-term care facilities," European Journal of Operational Research, vol. 206, no. 1, pp. 168-177, 2010.

[27] C. Toregas, R. Swain, C. ReVelle et al., "The location of emergency service facilities," Operations Research, vol. 19, no. 6, pp. 1363-1373, 1971.

[28] M. S. Daskin and E. H. Stern, "Hierarchical objective set covering model for emergency medical service vehicle deployment," Transportation Science, vol. 15, no. 2, pp. 137-152, 1981. 
[29] M. Daskin, "Network and discrete location: models, algorithms and applications," Journal of the Operational Research Society, vol. 48 , no. 7, pp. 763-763, 1997.

[30] V. Marianov and C. ReVelle, "The queueing maximal availability location problem: a model for the siting of emergency vehicles," European Journal of Operational Research, vol. 93, no. 1, pp. 110120, 1996.

[31] V. Verter and S. D. Lapierre, "Location of preventive health care facilities," Annals of Operations Research, vol. 110, no. 1-4, pp. 123-132, 2002.

[32] H. Jia, F. Ordonez, and M. Dessouky, "A modeling framework for facility location of medical services for large-scale emergencies," IIE Transactions, vol. 39, no. 1, pp. 41-55, 2007.

[33] P. Beraldi and M. E. Bruni, "A probabilistic model applied to emergency service vehicle location," European Journal of Operational Research, vol. 196, no. 1, pp. 323-331, 2009.

[34] H. O. Mete and Z. B. Zabinsky, "Stochastic optimization of medical supply location and distribution in disaster management," International Journal of Production Economics, vol. 126, no. 1, pp. 76-84, 2010.

[35] N. Coskun and R. Erol, "An optimization model for locating and sizing emergency medical service stations," Journal of Medical Systems, vol. 34, no. 1, pp. 43-49, 2010.

[36] Z. Yujun, C. Shengyong, and L. Haifeng, "Agent-based cooperative evolutionary computation for disaster rescue operation planning," Disaster Advances, vol. 5, no. 4, pp. 698-703, 2012.

[37] S. L. Hakimi, "Optimum locations of switching centers and the absolute centers and medians of a graph," Operations Research, vol. 12, no. 3, pp. 450-459, 1964.

[38] A. A. Kuehn and M. J. Hamburger, "A heuristic program for locating warehouses," Management Science, vol. 9, no. 4, pp. 643-666, 1963.

[39] R. Church and C. ReVelle, "The maximal covering location problem," Papers of the Regional Science Association, vol. 32, no. 1, pp. 101-118, 1974.

[40] M. S. Daskin, A. P. Hurter, and M. G. VanBuer, Toward an Integrated Model of Facility Location and Transportation Network Design, Working Paper, Transportation Center, Northwestern University, 1993.

[41] S. Melkote, Integrated models of facility location and network design [Doctor of philosophy], Northwestern University, Evaston, Ill, USA, 1996.

[42] S. Melkote and M. S. Daskin, "Capacitated facility location/network design problems," European Journal of Operational Research, vol. 129, no. 3, pp. 481-495, 2001.

[43] S. Melkote and M. S. Daskin, "An integrated model of facility location and transportation network design," Transportation Research A, vol. 35, no. 6, pp. 515-538, 2001.

[44] Z. Drezner and G. O. Wesolowsky, "Network design: selection and design of links and facility location," Transportation Research A, vol. 37, no. 3, pp. 241-256, 2003.

[45] C. Cocking, Solutions to facility location-network design problems [Doctor of philosophy thesis], Department of Computer Science, University of Heidelberg, 2008.

[46] C. Cocking and G. Reinelt, "Heuristics for budget facility location-network design problems with minisum objective," in Operations Research Proceedings 2008, Springer, Berlin, Germany, 2009.

[47] J. F. Bigotte, D. Krass, A. P. Antunes, and O. Berman, "Integrated modeling of urban hierarchy and transportation network planning," Transportation Research A, vol. 44, no. 7, pp. 506-522, 2010.
[48] L. Snyder and N. Ülker, "A model for locating capacitated, unreliable facilities," in Proceedings of the 14th Annual Industrial Engineering Research Conference (IERC '05), Atlanta, Ga, USA, May 2005.

[49] L. V. Snyder, Supply chain robustness and reliability: models and algorithms [Ph.D. thesis], Department of Industrial Engineering and Management Sciences, Northwestern University, Evanston, Ill, USA, 2003.

[50] L. V. Snyder and M. S. Daskin, "Reliability models for facility location: the expected failure cost case," Transportation Science, vol. 39, no. 3, pp. 400-416, 2005.

[51] O. Berman, D. Krass, and M. B. C. Menezes, "Facility reliability issues in network p-median problems: strategic centralization and co-location effects," Operations Research, vol. 55, no. 2, pp. 332-350, 2007.

[52] Z.-J. M. Shen, R. L. Zhan, and J. Zhang, The Reliable Facility Location Problem: Formulations, Heuristics, and Approximation Algorithms, Working Paper, University of California, Berkeley, Calif, USA, 2009.

[53] M. Lim, M. S. Daskin, A. Bassamboo, and S. Chopra, Facility Location Decisions in Supply Chain Networks with Random Disruption and Imperfect Information, Working Paper, Department of Business Administration, University of Illinois, 2009.

[54] L. Qi and Z. J. M. Shen, "A supply chain design model with unreliable supply," Naval Research Logistics, vol. 54, no. 8, pp. 829-844, 2007.

[55] L. Qi, Z. J. M. Shen, and L. V. Snyder, "The effect of supply disruptions on supply chain design decisions," Transportation Science, vol. 44, no. 2, pp. 274-289, 2010.

[56] L. V. Snyder and M. S. Daskin, "Models for reliable supply chain network design," in Critical Infrastructure: Reliability and Vulnerability, A. T. Murray and T. H. Grubesic, Eds., chapter 13, pp. 257-289, 2007.

[57] P. Peng, L. V. Snyder, A. Lim, and Z. Liu, "Reliable logistics networks design with facility disruptions," Transportation Research $B$, vol. 45, no. 8, pp. 1190-1211, 2011.

[58] A. Jabbarzadeh, S. G. Jalali Naini, H. Davoudpour, and N. Azad, "Designing a supply chain network under the risk of disruptions," Mathematical Problems in Engineering, vol. 2012, Article ID 234324, 23 pages, 2012.

[59] H. A. Makse, S. Havlin, and H. E. Stanley, "Modelling urban growth patterns," Nature, vol. 377, no. 6550, pp. 608-612, 1995.

[60] Z. Wu, L. A. Braunstein, S. Havlin, and H. E. Stanley, "Transport in weighted networks: partition into superhighways and roads," Physical Review Letters, vol. 96, no. 14, Article ID 148702, 4 pages, 2006.

[61] K. Gangopadhyay and B. Basu, "City size distributions for India and China," Physica A, vol. 388, no. 13, pp. 2682-2688, 2009.

[62] T. Musha, H. Takeuchi, and T. Inoue, "1/f fluctuations in the spontaneous spike discharge intervals of a giant snail neuron," IEEE Transactions on Biomedical Engineering, vol. 30, no. 3, pp. 194-197, 1983.

[63] B. Neumcke, "1/f noise in membranes," Biophysics of Structure and Mechanism, vol. 4, no. 3, pp. 179-199, 1978.

[64] M. Li and W. Zhao, "On 1/f noise," Mathematical Problems in Engineering, vol. 2012, Article ID 673648, 23 pages, 2012.

[65] C. Cattani, "On the existence of wavelet symmetries in Archaea DNA," Computational and Mathematical Methods in Medicine, vol. 2012, Article ID 673934, 21 pages, 2012.

[66] C. Cattani, G. Pierro, and G. Altieri, "Entropy and multifractality for the myeloma multiple TET 2 gene," Mathematical 
Problems in Engineering, vol. 2012, Article ID 193761, 14 pages, 2012.

[67] C. Cattani, "Fractals and hidden symmetries in DNA," Mathematical Problems in Engineering, vol. 2010, Article ID 507056, 31 pages, 2010.

[68] M. Li and W. Zhao, "On bandlimitedness and lag-limitedness of fractional Gaussian noise," Physica A, vol. 392, no. 9, pp. 19551961, 2013.

[69] X. Zhao, P. J. Shang, A. Lin, and G. Chen, "Multifractal Fourier detrended cross-correlation analysis of traffic signals," Physica A, vol. 390, no. 21-22, pp. 3670-3678, 2011.

[70] N. A. Krüger, "Estimating traffic demand risk-a multiscale analysis," Transportation Research A, vol. 46, no. 10, pp. 17411751, 2012.

[71] L. M. Zwack, C. J. Paciorek, J. D. Spengler, and J. I. Levy, "Characterizing local traffic contributions to particulate air pollution in street canyons using mobile monitoring techniques," Atmospheric Environment, vol. 45, no. 15, pp. 2507-2514, 2011. 


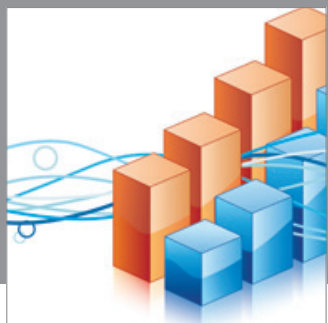

Advances in

Operations Research

mansans

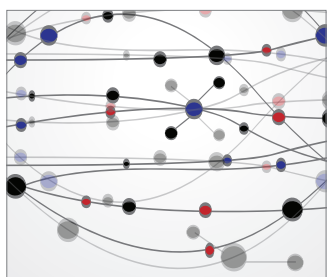

The Scientific World Journal
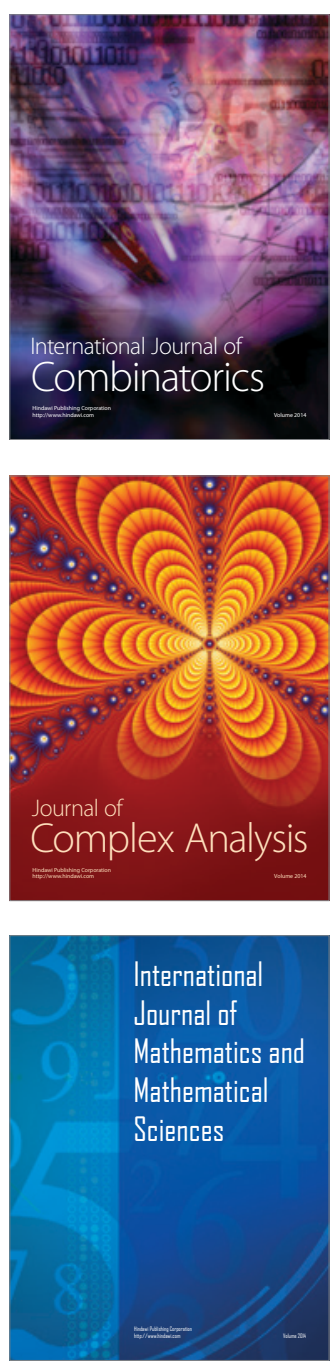
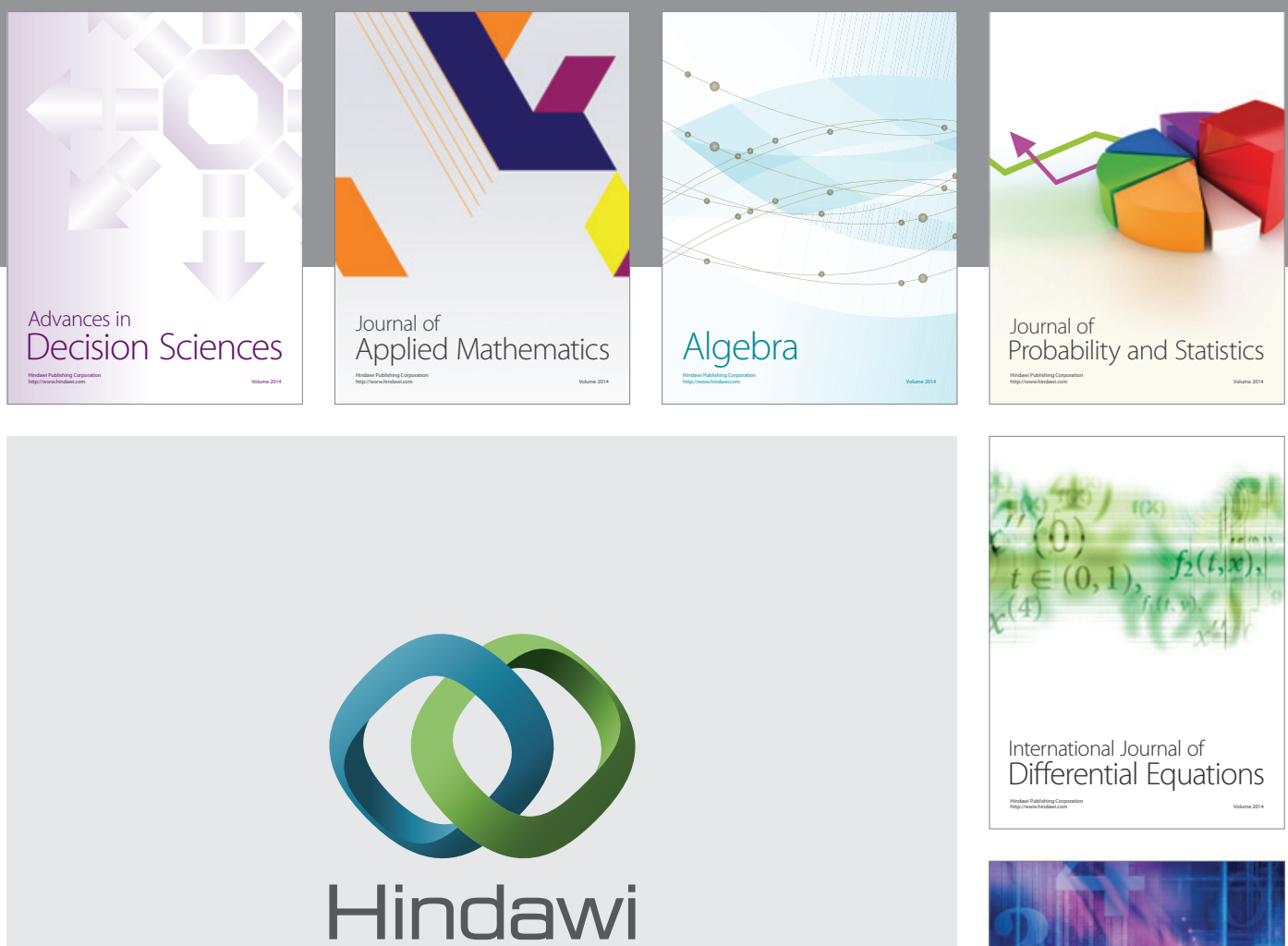

Submit your manuscripts at http://www.hindawi.com
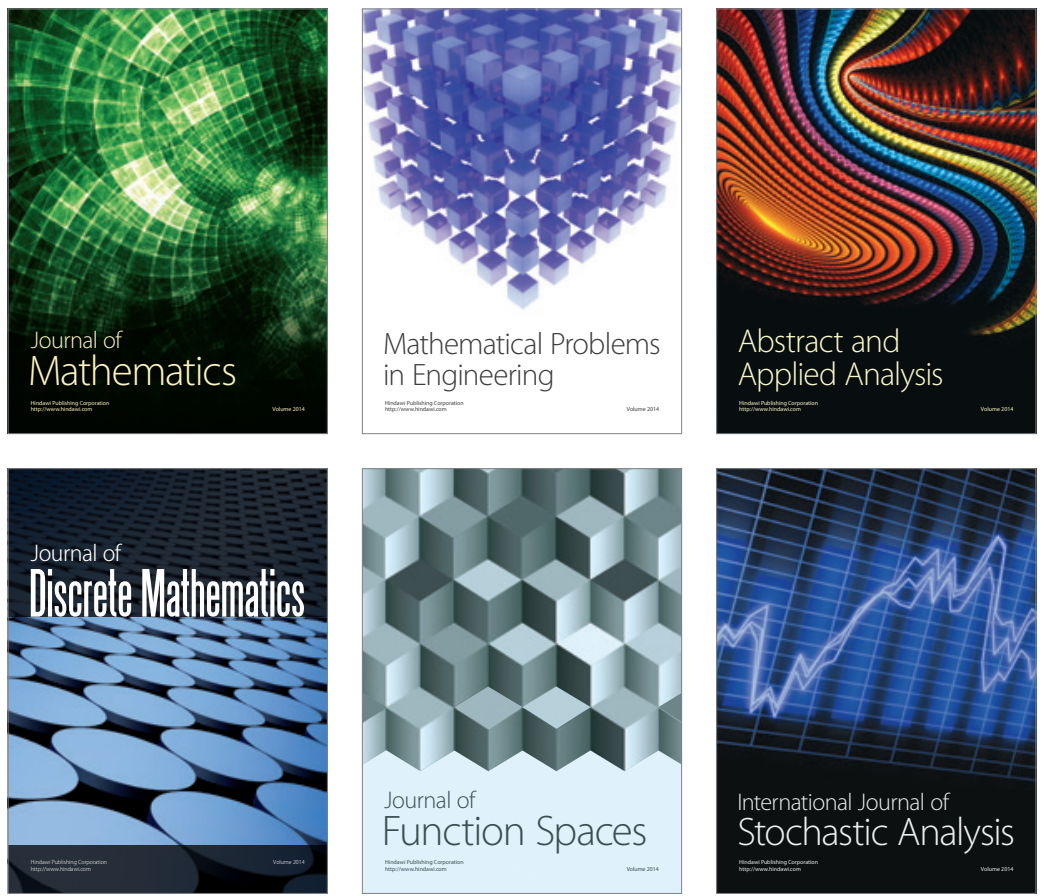

Journal of

Function Spaces

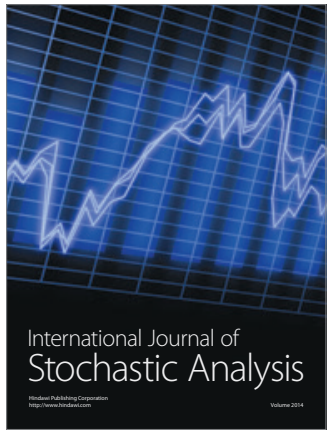

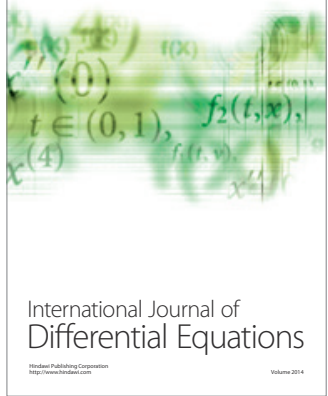
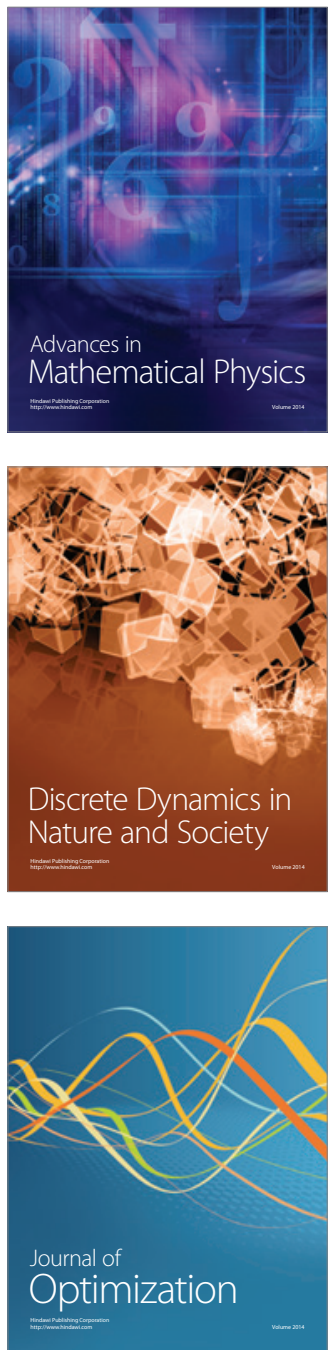